\title{
EVA BAYER-FLUCKIGER
}

\section{Réseaux unimodulaires}

Séminaire de Théorie des Nombres de Bordeaux, tome 1, no 1 (1989), p. $189-196$

<http://www.numdam.org/item?id=JTNB_1989_1_1_189_0>

(C) Université Bordeaux 1, 1989, tous droits réservés.

L'accès aux archives de la revue "Séminaire de Théorie des Nombres de Bordeaux » (http://jtnb.cedram.org/) implique l'accord avec les conditions générales d'utilisation (http://www.numdam.org/conditions). Toute utilisation commerciale ou impression systématique est constitutive d'une infraction pénale. Toute copie ou impression de ce fichier doit contenir la présente mention de copyright.

\section{Numdam}

Article numérisé dans le cadre du programme

Numérisation de documents anciens mathématiques

http://www.numdam.org/ 
Séminaire de Théorie des Nombres,

Bordeaux, 1,189-196(1989)

\title{
Réseaux unimodulaires.
}

\author{
par Eva BAYER-FLUCKIGER
}

\begin{abstract}
Résumé - Soit $f$ un produit de polynômes cyclotomiques. Existe-t-il une forme bilinéaire symétrique entière, unimodulaire et définie positive ayant une isométrie de polynôme caractéristique $f$ ? Ce travail donne une réponse partielle à cette question.
\end{abstract}

Abstract - Let $f$ be a product of cyclotomic polynomials. Does there exist an integral, unimodular and positive definite symmetric bilinear form that has an isometry with characteristic polynomial $f$ ? The present paper gives a partial answer to this question.

\section{1 - Isométries parfaites}

Le point de départ de ce travail est une question de Claude Weber, motivée par la théorie des noeuds [4], [5] :

Soit $L$ un Z-module libre de rang $n$, et soit $S: L \times L \rightarrow \mathbf{Z}$ une forme bilinéaire $\epsilon$-symétrique $(\epsilon=1$ ou -1$)$ unimodulaire et paire (c'est-à-dire $S(x, x) \equiv 0(\bmod 2)$ pour tout $x \in L)$.

Question 1:

Existe-t-il une forme bilinéaire unimodulaire $A: L \times L \rightarrow \mathbf{Z}$ telle que $S(x, y)=A(x, y)+\epsilon A(y, x)$ pour tout $x, y \in L$ ?

Michel Kervaire a étudié cette question [5]. Lorsque $\epsilon=-1$, la réponse est affirmative. Si $\epsilon=1$ et $S$ est indéfinie, Kervaire a montré que la réponse est encore positive sauf si $n=2$. Il a utilisé pour cela la classification des formes bilinéaires symétriques entières, unimodulaires et indéfinies [6], [9]. Pour aborder le cas où $S$ est symétrique et défini, il a introduit la notion d'isométrie parfaite :

DÉfinition. Un isomorphisme de Z-modules $t: L \rightarrow L$ est une isométrie de $S$ si $S(t x, t y)=S(x, y)$ pour tout $x, y \in L$. Une isométrie $t: L \rightarrow L$ de $S$ est appelée isométrie parfaite si $(1-t): L \rightarrow L$ est un isomorphisme.

Manuscrit reçu le 16 mai 1989. 
Lemme 1. [5] Il existe une forme bilinéaire unimodulaire $A: L \times L \rightarrow \mathbf{Z}$ telle que $S(x, y)=A(x, y)+\epsilon A(y, x)$ pour tout $x, y \in L$ si et seulement si $S$ a une isométrie parfaite.

\section{DÉMONSTRATION :}

Etant donné $A$, on définit $t: L \rightarrow L$ par

$$
A(t x, y)=-\epsilon A(y, x) .
$$

On vérifie que $t$ est bien une isométrie de $S$, et que $S(x, y)=A((1-t) x, y)$. Comme $A$ est unimodulaire, $1-t$ est un isomorphisme. Réciproquement, si $t$ est une isométrie parfaite de $S$, posons $A(x, y)=S\left((1-t)^{-1} x, y\right)$. On vérifie que $S(x, y)=A(x, y)+\epsilon A(y, x)$. Une démonstration détaillée se trouve dans [5], page 178 .

\section{Remarque}

Le lemme et sa démonstration sont inspirés par la théorie des noeuds [5].

On peut donc reformuler la question 1 comme suit :

\section{Question 2:}

Est-ce que $S$ admet une isométrie parfaite?

Supposons désormais $S$ symétrique et définie positive. Comme $S$ est paire, il est bien connu que le rang de $S$ est divisible par 8 (voir par exemple [9], chapitre V, Corollaire 2). Rappelons qu'il existe une unique classe d'isomorphisme de formes bilinéaires symétriques unimodulaires de rang 8 , notée $\Gamma_{8}$. Il y en a deux de rang 16 , une indécomposable notée $\Gamma_{16}$ et la somme orthogonale de deux copies de $\Gamma_{8}$. Pour $n=24$, Niemeier a démontré qu'il existe exactement 24 classes d'isomorphisme de telles formes [7]. L'une de ces formes, appelée forme de Leech, a une propriété particulière :

Définition. Le minimum de $S$ est le plus petit entier non nul $m$ tel qu'il existe $x \in L$ avec $S(x, x)=m$.

La forme de Leech est de minimum 4, alors que les formes de Niemeier sont de minimum 2.

DÉfinition. On appelle réseau une forme bilinéaire symétrique entière et définie positive. 
Kervaire [5] a donné une réponse complète à la Question 2 pour les réseaux de rang au plus 24 . Le réseau $\Gamma_{8}$ a une isométrie parfaite, $\Gamma_{16}$ n'en a pas. Parmi les réseaux unimodulaires de rang 24,10 ont une isométrie parfaite (dont le réseau de Leech), 14 n'en ont pas. Kervaire a aussi obtenu des résultats concernant des réseaux de rang arbitrairement grand et de minimum 2 (voir [5]).

On dit qu'un réseau est indécomposable s'il n'est pas isomorphe à une somme orthogonale de réseaux non triviaux. Le résultat suivant est une conséquence du Théorème 1 et de la Proposition 2 (voir 2) :

Proposition 1. Il existe une infinité de classes d'isomorphisme de réseaux unimodulaires, indécomposables et de minimum au moins 4 qui ont une isométrie parfaite.

Soit $(L, S)$ un réseau et soit $t: L \rightarrow L$ une isométrie de $S$. Soit $f$ le polynôme caractéristique de $t$. Alors $t$ est parfaite si et seulement si $f(1)= \pm 1$. Au lieu de chercher des réseaux ayant une isométrie parfaite, on peut se poser une question plus précise et s'intéresser aux réseaux ayant une isométrie de polynôme caractéristique donné. Ceci fera l'objet du paragraphe suivant.

2. Réseaux unimodulaires ayant une isométrie de polynôme caractéristique donné

Soit $f$ un polynôme unitaire à coefficients entiers. Un $f$-réseau est un réseau ayant une isométrie de polynôme caractéristique $f$. Le groupe des isométries d'un réseau est fini. Donc s'il existe un $f$-réseau, alors $f$ est un produit de polynômes cyclotomiques. Notons $\phi_{m}$ le polynôme cyclotomique correspondant aux racines $m^{\text {ièmes }}$ de l'unité.

THÉORÈmE 1 [2]. :

Soit $f=\phi_{m}^{n}$. Alors

1) Si $m$ est une puissance de 2, alors il existe un $f$-réseau unimodulaire.

2) Si $m$ n'est pas une puissance de 2, alors il existe un $f$ - réseau unimodulaire si et seulement si le degré de $f$ est divisible par 8 , et $f(1) f(-1)$ est un carré. 


\section{Proposition 2 [2].}

Soit $f=\phi_{m}$, avec $m$ sans facteur carré. Alors tout $f$-réseau est indécomposable. Si $m$ n'est pas un nombre premier et si $\operatorname{deg}(f)>8$, alors tout $f$-réseau est de minimum au moins 4 .

\section{Démonstration de la Proposition 1:}

Soit $m=p q r$, avec $p, q$ et $r$ des nombres premiers impairs distincts. Posons $f=\phi_{m}$. Alors $\operatorname{deg}(f)=(p-1)(q-1)(r-1)$, donc $\operatorname{deg}(f)$ est divisible par 8. On a $f(1)=f(-1)=1$. Les conditions du théorème 1 sont remplies, il existe donc un $f$-réseau unimodulaire $(L, S)$. Soit $t: L \rightarrow L$ une isométrie de $S$ de polynôme caractéristique $f$. On a $f(1)=1$, donc $t$ est parfaite. La proposition 2 entraîne que $(L, S)$ est indécomposable et de minimum au moins 4 .

On dit que deux polynômes $f, g \in \mathbf{Z}[X]$ sont premiers entre eux s'il existe $h, k \in \mathbf{Z}[X]$ avec $f h+g k=1$.

Lemme 2 ([2], Remark (1.3)).

La somme orthogonale d'un $f$-réseau et d'un $g$-réseau est un $(f g)-$ réseau. Réciproquement, si $f$ et $g$ sont premiers entre eux alors tout $(f g)$ réseau se décompose en la somme orthogonale d'un $f$-réseau et d'un $g$ réseau.

Il est bien connu que $\phi_{n}$ et $\phi_{m}$ ne sont pas premiers entre eux si et seulement si $n=p^{r} m$, où $p$ est un nombre premier et $r$ un nombre entier. Par exemple, $\phi_{p^{n}}$ et $\phi_{p^{m}}$ ne sont pas premiers entre eux si $p$ est un nombre premier. Le théorème 1 montre que si $p$ est un nombre premier impair, alors il n'existe pas de $\phi_{p^{n}}$ - réseau unimodulaire.

Proposition 3. Soit $p$ un nombre premier impair, et posons $f=\phi_{p^{n}} \phi_{p^{m}}$ où $n$ et $m$ sont des nombres entiers positifs. Les propriétés suivantes sont équivalentes :

(a) Il existe un $f$-réseau unimodulaire

(b) $\operatorname{deg}(f)$ est divisible par 8

(c) $p \equiv 1(\bmod 4)$ ou $n+m \equiv 1(\bmod 2)$.

DÉmonstration: L'équivalence de $(b)$ et de $(c)$ résulte de propriétés élémentaires des polynômes cyclotomiques. $\mathrm{Si}(L, S)$ est un $f$-réseau unimodulaire, alors $S$ est pair (cf. [2], démonstration du lemme (1.4)). Donc le 
rang de $L$ est divisible par 8. Mais $\operatorname{rang}(L)=\operatorname{deg}(f)$. Donc (a) entraîne (b). Il reste à montrer que (c) entraîne (a). La démonstration de ce fait sera donnée dans le 5 , après quelques préliminaires sur les formes de torsion.

\section{3 - Formes de torsion}

Soit $C$ un groupe (dans la suite, nous prendrons pour $C$ le groupe trivial ou le groupe cyclique infini $C_{\infty}$ ). Une forme Z- bilinéaire symétrique $S$ : $G \times G \rightarrow \mathbf{Q} / \mathbf{Z}$ sur un groupe abélien fini $G$ est non dégénérée si l'homomorphisme $\hat{S}: G \rightarrow \operatorname{Hom}_{Z}(G, \mathbf{Q} / \mathbf{Z})$, défini par $\hat{S}(x)(y)=S(x, y)$, est un isomorphisme. Si on a de plus $S(t x, t y)=S(x, y)$ pour tout $x, y \in G$ et $t \in C$, alors $(G, S)$ est appelée une $C$-forme de torsion. La somme orthogonale de deux formes de torsion $(G, S)$ et $\left(G^{\prime}, S^{\prime}\right)$ est par définition $\left(G \oplus G^{\prime}, S \oplus S^{\prime}\right)$, où $\left(S \oplus S^{\prime}\right)\left(x+x^{\prime}, y+y^{\prime}\right)=S^{\prime}\left(x^{\prime}, y^{\prime}\right)$. Si $t \in C$, on pose $t\left(x, x^{\prime}\right)=\left(t x, t x^{\prime}\right)$.

Soit $(G, S)$ une forme de torsion. Pour tout sous-groupe $H$ de $G$, posons $H^{\perp}=\{x \in G \mid S(x, y)=0$ pour tout $y \in H\}$. On dit que $(G, S)$ est neutre s'il existe un sous-groupe $U$ de $G$ tel que $U^{\perp}=U$, et $t(U)=U$ pour tout $t \in C$. Deux formes de torsion $(G, S)$ et $\left(G^{\prime}, S^{\prime}\right)$ sont isomorphes s'il existe un isomorphisme de $Z$-modules $f: G \rightarrow G^{\prime}$ tel que $t$ o $f=f \circ t$ pour tout $t \in C$, et $S^{\prime}(f x, f y)=S(x, y)$ pour tout $x, y \in G$.

Soit $G(\mathbf{Q} / \mathbf{Z}, C)$ le groupe de Grothendieck des classes d'isomorphisme des $C$-formes de torsion par rapport à la somme orthogonale. Soit $W(\mathbf{Q} / \mathbf{Z}, C)$ le quotient de $G(\mathbf{Q} / \mathbf{Z}, C)$ par le sous-groupe engendré par les formes neutres. Ce groupe est appelé groupe de Witt des $C$-formes de torsion. Si $C$ est le groupe trivial, on pose $W(\mathbf{Q} / \mathbf{Z}, C)=W(\mathbf{Q} / \mathbf{Z})$. On $\operatorname{dit}$ que deux formes de torsion sont équivalentes si elles sont dans la même classe de $W(\mathbf{Q} / \mathbf{Z}, C)$. Il est facile de vérifier que $(G, S)$ et $\left(G^{\prime}, S^{\prime}\right)$ sont équivalents si et seulement s'il existe des formes neutres $(N, T)$ et $\left(N^{\prime}, T^{\prime}\right)$ telles que $(G, S) \oplus(N, T) \tilde{=}\left(G^{\prime}, S^{\prime}\right) \oplus\left(N^{\prime}, T^{\prime}\right)$.

Le lemme suivant est bien connu :

Lem 3 [8], (5.1.3). Soit $(G, S)$ une forme de torsion, et soit $H$ un sousgroupe de $G$ tel que $H \subset H^{\perp}$ et $t(H)=H$ pour tout $t \in C$. Alors $(G, S)$ et $\left(H^{\perp} / H, S\right)$ sont équivalentes.

\section{Construction de $f$-réseaux unimodulaires}

Soit $(M, S)$ un $f$-réseau, et soit $t: M \rightarrow M$ une isométrie de $S$ de polynôme caractéristique $f$. Soit $V=M \otimes_{\mathbf{Z}} \mathbf{Q}$. On obtient $S: V \times V \rightarrow \mathbf{Q}$ et $t: V \rightarrow V$ par extension des scalaires. Posons $M^{\prime}=\{x \in V \mid S(x, y) \in \mathbf{Z}$ 
pour tout $y \in M\}$. On a $t\left(M^{\prime}\right)=M^{\prime}$. Soit $G=M^{\prime} / M$. On obtient une forme symétrique $S: G \times G \rightarrow \mathbf{Q} / \mathbf{Z}$ et une isométrie $t: G \rightarrow G$ de cette forme. Alors $(G, S)$ est une $C=C_{\infty}$-forme de torsion. Supposons que $(G, S)$ soit neutre. Il existe alors un sous-groupe $U$ de $G$ tel que $U^{\perp}=U$ et $t(U)=U$. Notons $p: M^{\prime} \rightarrow G=M^{\prime} / M$ la projection. Soit $L=p^{-1}(U)$. Alors $L^{\prime}=L$ et $t(L)=L$. Donc $L$ est un $f$-réseau unimodulaire.

\section{Démonstration de la Proposition 3}

Soit $\left(e_{1}, \cdots, e_{p}\right)$ la base standard de $\mathbf{R}^{p}$. On munit $\mathbf{R}^{p}$ du produit scalaire canonique $e_{i} \cdot e_{j}$. Soit

$$
A=\mathbf{Z} A_{p-1}=\left\{\sum a_{i} e_{i} \mid a_{i} \in \mathbf{Z} \quad \sum a_{i}=0\right\}
$$

le réseau de rang $p-1$ engendré par le système de racines $A_{p-1}$ (voir par exemple [7], 4). On a $A^{\prime} / A \tilde{=} \mathbf{Z} / p \mathbf{Z}$. Notons $r A$ la somme orthogonale de $r$ copies de $A$. Posons $M_{n}=p^{n-1} A$. La base canonique du $\mathbf{R}^{p}$ contenant la $j$ ème copie de $A$ sera notée $\left(e_{1}^{j}, \cdots e_{p}^{j}\right)$. Soit $t_{n}: M_{n} \rightarrow M_{n}$ l'isométrie de $M_{n}$ définie par

$$
t_{n}\left(e_{i}^{j}\right)=e_{i}^{j+1} \text { si } j \neq p^{n-1}, t_{n}\left(e_{i}^{p^{n-1}}\right)=e_{i+1}^{1}
$$

où $i$ est un indice modulo $p$. Alors le polynôme caractéristique de $t_{n}$ est $\phi_{p^{n}}$. Soit $M$ la somme orthogonale de $M_{n}$ et de $M_{m}$, muni de l'isométrie $t=t_{n} \oplus t_{m}$. Alors $M$ est un $\phi_{p^{n}} \phi_{p^{m} \text {-réseau. }}$

Posons $G_{n}=M_{n}^{\prime} / M_{n}$. La $C_{\infty}$-forme de torsion $\left(G_{n}, S_{n}\right)$ associée à $M_{n}$ est la somme orthogonale de $p^{n-1}$ copies de

$$
\begin{gathered}
T: \mathbf{Z} / p \mathbf{Z} \times \mathbf{Z} / p \mathbf{Z} \rightarrow \mathbf{Q} / \mathbf{Z} \\
T(x, y)=-\frac{1}{p} x y
\end{gathered}
$$

(voir [7], §4), munie de l'isométrie $t_{n}: G_{n} \rightarrow G_{n}$.

Dans $W(\mathbf{Q} / \mathbf{Z})$, la somme orthogonale de 4 copies de $T$ est toujours nulle, et celle de 2 copies de $T$ l'est si et seulement si $p \equiv 1(\bmod 4)$. Donc dans $W(\mathbf{Q} / \mathbf{Z})$, la classe de la forme de torsion $\left(G_{n}, S_{n}\right)$ est égale à celle de

$$
\begin{gathered}
T_{n}=\mathbf{Z} / p \mathbf{Z} \times \mathbf{Z} / p \mathbf{Z} \rightarrow \mathbf{Q} / \mathbf{Z} \\
T_{n}(x, y)=-\frac{\epsilon}{p} x y
\end{gathered}
$$


où $\epsilon \equiv p^{n-1}(\bmod 4)$.

Remarquons que la $C_{\infty}$ - forme de torsion $\left(G_{n}, S_{n}\right)$ s'identifie à l'anneau de groupe $\mathrm{F}_{p} C_{p^{n-1}}$ (où $\mathrm{F}_{p}$ est le corps fini à $p$ éléments et $C_{r}$ est le groupe cyclique d'ordre $r$ ) muni de l'opposée de la forme standard. Soit $I=$ $\left(t_{n}-1\right)\left(G_{n}\right)$, l'idéal d'augmentation de cet anneau de groupe. Posons $k=\frac{n-1}{2}$, et $U=I^{k}$. Alors $U \subset U^{\perp}=I^{k-1}$. Par le lemme 3, les $C_{\infty}$-formes de torsion $\left(G_{n}, S_{n}\right)$ et $\left(U^{\perp} / U, S_{n}\right)$ sont équivalentes. On a $U^{\perp} / U \cong \mathbf{Z} / p \mathbb{Z}$ et l'isométrie induite par $t_{n}$ est l'identité. Donc $\left(G_{n}, S_{n}\right)$ est équivalente à $T_{n}$ en tant que $C_{\infty}$-formes de torsion.

Soit $(G, S)=\left(G_{n}, S_{n}\right) \oplus\left(G_{m}, S_{m}\right)$ la $C_{\infty}$-forme de torsion associée à $M$. Cette forme est donc équivalente à $T_{n} \oplus T_{m}$. Si $p \equiv 1(\bmod 4), T_{n} \oplus T_{m}$ est neutre. Si $p \equiv 3(\bmod 4)$, alors $T_{n} \oplus T_{m}$ est neutre si et seulement si $n+m$ est impair. Donc l'hypothèse (c) entraîne que $(G, S)$ est neutre. La construction du 4 nous fournit donc un $\phi_{p^{n}} \phi_{p^{m}}$-réseau unimodulaire.

\section{Remarque}

Au cours d'une étude de la forme trace d'une extension cyclique d'ordre $p^{2}$ dans laquelle $p$ est totalement ramifié [1], Christine Bachoc et Boas Erez obtiennent des $\phi_{p} \phi_{p^{2}}$-réseaux unimodulaires. Le système des racines de

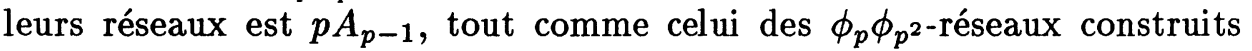
dans la démonstration ci-dessus.

\section{BiBLIOGRAPHIE}

[1] C. BACHOC et B. EREZ Forme trace et ramification. (à paraître).

[2] E. BAYER-FLUCKIGER Definite unimodular lattices having an automorphism of given characteristic polynomial, Comment. Math. Helv. 59 (1984), 509-538.

[3] E. BAYER-FLUCKIGER Unimodular lattices. (en préparation).

[4] J-C. HAUSMAN (éditeur) Problems in knot theory., Proceedings of a conference on knot theory, Plans-sur-Bex 1977 lecture Notes Math 685. Springer Verlag, 1978, 309-311.

[5] M. KERVAIRE Formes de Seifert et fromes quadratiques entières, L'enseignement Math. 31 (1985), 173-186.

[6] J. MILNOR, D. HUSEMOLLER Symetric bilinear forms, Ergebnisse Math. 73. Springer Verlag (1973).

[7] H-V. NIEMEIER Definite quadratische Formen der Diskriminante 1 und Dimension 24, J. Number Theory 5 (1973), 142-178.

[8] W. SCHARLAU Quadratic and hermitian forms, Grundlehren Math. Wiss. 270. Springer Verlag (1985). 
[9] J-P. SERRE Cours d'arithmétique, Presses Universitaires de France (1970).

Mots clefs: Formes quadratiques entières.

U.A. 741 du C.N.R.S.

Laboratoire de Mathématiques

Faculté des Sciences de Besançon

25030 Besançon Cedex, FRANCE. 